\title{
Iron accelerates hemoglobin oxidation increasing mortality in vascular diseased guinea pigs following transfusion of stored blood
}

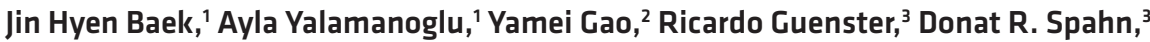 \\ Dominik J. Schaer, ${ }^{4}$ and Paul W. Buehler ${ }^{1}$ \\ 'Laboratory of Biochemistry and Vascular Biology, ${ }^{2}$ Division of Viral Products, Center of Biologics Evaluation and Research \\ (CBER), FDA, Silver Spring, Maryland, USA. ${ }^{3}$ Institute of Anesthesiology, University of Zurich and University Hospital of \\ Zurich, Zurich, Switzerland. ${ }^{4}$ Division of Internal Medicine, University of Zurich, Zurich, Switzerland.
}

\begin{abstract}
Non-transferrin-bound iron (NTBI) and free hemoglobin ( $\mathrm{Hb}$ ) accumulate in circulation following stored RBC transfusions. This study investigated transfusion, vascular disease, and mortality in guinea pigs after stored RBC transfusion alone and following cotransfusion with apo-transferrin (apo-Tf) and haptoglobin (Hp). The effects of RBC exchange transfusion dose (1, 3, and 9 units), storage period (14 days), and mortality were evaluated in guinea pigs with a vascular disease phenotype. Seven-day mortality and the interaction between iron and $\mathrm{Hb}$ as cocontributors to adverse outcome were studied. Concentrations of iron and free $\mathrm{Hb}$ were greatest after transfusion with 9 units of stored RBCs compared with fresh RBCs or stored RBCs at 1- and 3-unit volumes. Nine units of stored RBCs led to mortality in vascular diseased animals, but not normal animals. One and 3 units of stored RBCs did not cause a mortality effect, suggesting the concomitant relevance of NTBI and $\mathrm{Hb}$ on outcome. Cotransfusion with apo-Tf or Hp restored survival to $100 \%$ following 9-unit RBC transfusions in vascular diseased animals. Our data suggest that increases in plasma NTBI and $\mathrm{Hb}$ contribute to vascular disease-associated mortality through iron-enhanced $\mathrm{Hb}$ oxidation and enhanced tissue injury.
\end{abstract}

Conflict of interest: D.R. Spahn receives honoraria and travel support from several private and publicly held companies (list of companies provided in the online supplement).

Submitted: February 21, 2017

Accepted: April 4, 2017

Published: May 4, 2017

Reference information: JCI Insight. 2017;2(9):e93577. https:// doi.org/10.1172/jci.insight.93577.

\section{Introduction}

Millions of patients receive blood transfusions worldwide, with nearly 15 million units collected in the U.S. alone. Of the units collected, approximately one-fifth of RBCs remain in storage for as long as 4 to 6 weeks (1), making the safety of these storage units difficult to evaluate in humans (2) and prompting the U.S. NIH to reduce maximum FDA allowable storage limits from 42 days to 35 days (3). Several retrospective and prospective studies have assessed organ injury or morbidity, length of hospital stay, number of ventilator days, and all-cause mortality in patients transfused with blood after different storage periods (4-8). While some of these retrospective studies reported worse clinical outcome associated with transfusion of blood after a longer storage period $(4,5)$, another large cohort study found only a marginal increase in 7-day mortality after stored blood transfusion, which disappeared when the analysis was restricted to leukoreduced RBCs (9). Prospective clinical trials in different patient populations have also not confirmed the initial findings of increased morbidity and mortality after stored RBC transfusion $(6,7)$ These randomized trials reported that older stored blood compared with fresh blood did not alter clinical outcomes $(6,7,10)$ or surrogate markers of tissue oxygenation (11) in patients undergoing cardiac surgery as well as in critically ill adults and infants.

Preclinical models provide a unique opportunity to evaluate potential adverse effects of transfusion with RBC units approaching the end of the allowed storage period. Such data may not otherwise be obtainable from human trials. While preclinical studies cannot provide quantitative estimates of the clinical risk in different human transfusion scenarios, they can reveal insight into pathophysiological mechanisms of $\mathrm{RBC}$ transfusion-related toxicity and enhance understanding of the relationship between storage time, $\mathrm{RBC}$ units dosed, and underlying disease. 
When they occur, pathological responses to the transfusion of blood are complex, multifactorial, and complicated by comorbidities such as vascular disease. In animals the unintended consequences of RBC transfusion may be associated with extravascular hemolysis leading to non-transferrin-bound iron (NTBI) accumulation (12), intravascular hemolysis leading to release of free hemoglobin ( $\mathrm{Hb})(13)$, and ischemia reperfusion injury (14). In animals, these processes are reported to deregulate vascular nitric oxide (NO) homeostasis (15-17), promote inflammation (12), and exacerbate infection $(18,19)$. In humans, posttransfusion saturation of transferrin (Tf) increases and NTBI accumulates in plasma as a result of extravascular hemolysis, taking place in the spleen and liver $(20,21)$. As the number of RBC units administered increases, depletion of haptoglobin ( $\mathrm{Hp}$ ) levels leads to accumulation of cell-free $\mathrm{Hb}$ in plasma (22). Together, iron and $\mathrm{Hb}$ may lead to undesirable effects reported to occur following transfusion of older storage time blood.

In the present study, we hypothesized that transfusion of stored RBCs could increase mortality in guinea pigs with a diet-induced vascular disease phenotype and that outcome may be determined by both iron and $\mathrm{Hb}$ accumulation. We further studied if neutralization of iron and $\mathrm{Hb}$ by administration of the binding proteins Tf (as apo-Tf) and Hp, respectively, could be used to improve survival in our model. Herein we present data to support that neutralization of iron and cell-free $\mathrm{Hb}$ improves outcome following transfusion of stored guinea pig RBCs. Our data suggest that increases in plasma NTBI and Hb contribute to vascular disease-associated mortality though iron-enhanced $\mathrm{Hb}$ oxidation and subsequent tissue injury.

\section{Results}

Guinea pig model of preexisting vascular disease: vascular remodeling and endothelial dysfunction. Compared with normal diet (ND), our high-fat and high-sucrose diet (HFSD) guinea pigs developed structural changes in elastic arteries, characterized by increased collagen in medial and adventitial layers of aorta sections stained with Masson's trichrome (Figure 1A). In addition, HFSD guinea pigs demonstrated a fragmented pattern or complete loss of elastin fibers with increased collagen deposition in VerhoeffVan Gieson-stained aorta tissue sections (Figure 1A). The ratio of collagen to elastin was evaluated to determine the extent of remodeling within the aorta. The data demonstrate a 2.5 -fold increase in aortic collagen $(39.2 \% \pm 3.78 \%)$ in HFSD compared with ND $(15.3 \% \pm 4.87 \%)$ guinea pigs (Figure $1 \mathrm{~B})$ and a 7-fold increase in the aortic collagen to elastin ratio following 3 months of HFSD $(1.86 \pm 0.496)$ compared with ND $(0.300 \pm 0.127)$ in guinea pigs (Figure $1 \mathrm{C})$. The loss of elastin or its fragmentation as well as collagen deposition are critical components of vascular dysfunction and increased blood vessel stiffness, particularly in the central elastic vessels (23).

To measure the effect of a HFSD on vascular function, blood pressure was measured from an indwelling catheter placed in the common carotid artery. In all HFSD guinea pigs, baseline systolic and pulse pressures were consistently elevated compared with ND animals (Figure 1, D and E). Additionally, sequential injections of acetylcholine (Ach) were made over a dosing range from 0 to $10 \mu \mathrm{g}$ to ND and HFSD guinea pigs. Systolic and diastolic blood pressures were allowed to return to basal values prior to each subsequent Ach dose, consistent with endothelial dysfunction. Ach at each dosing level was consistently less vasodilatory in HFSD animals $(-15.7 \% \pm 7.74 \%[2.50 \mu \mathrm{g}],-21.4 \% \pm 8.39 \%[5$ $\mu \mathrm{g}]$, and $-28.0 \% \pm 6.91 \%[10 \mu \mathrm{g}])$ compared with ND animals $(-56.0 \% \pm 12.3 \%[2.50 \mu \mathrm{g}],-55.5 \% \pm$ $6.67 \%[5 \mu \mathrm{g}]$, and $-69.7 \% \pm 7.23 \%[10 \mu \mathrm{g}]$ ) (Figure 1F). Similar to diabetic mice $(24,25)$, HFSD guinea pigs demonstrated increased systemic blood pressure following stored-RBC (S-RBC; stored for 14 days) compared with fresh-RBC (F-RBC; used within 1 day of collection) transfusion. The blood pressure response to S-RBC transfusion was blunted following coadministration of high-dose $\mathrm{Hp}(300 \mathrm{mg} / \mathrm{kg})$, suggesting that increased $\mathrm{Hb}$ induced vasoactivity despite endothelial dysfunction, also similar to diabetic mice. This hemodynamic response in HFSD guinea pigs was associated with $\mathrm{Hb}$-induced expression of inactive eNOS (Supplemental Figure 1; supplemental material available online with this article; https://doi.org/10.1172/jci.insight.93577DS1). These characteristics mimic a frequent cardiovascular comorbidity found in patients undergoing blood transfusion (26).

Transfusion of stored guinea pig RBCs leads to dose-dependent accumulation of plasma iron and cell-free $\mathrm{Hb}$. Storage parameters and posttransfusion survival were tested for F-RBCs and S-RBCs (Table 1). Data presented in Table 1 show the mean \pm SD $(95 \%$ CI) differences in guinea pig RBC parameters following overnight storage (day 1) compared with storage for 14 days. In the present study blood, was collected in anticoagulant citrate phosphate double dextrose (CP2D) and RBCs were stored in additive solution formula 3 (AS-3). The trends in RBC parameter changes for human blood after collection in CP2D and 
A

ND
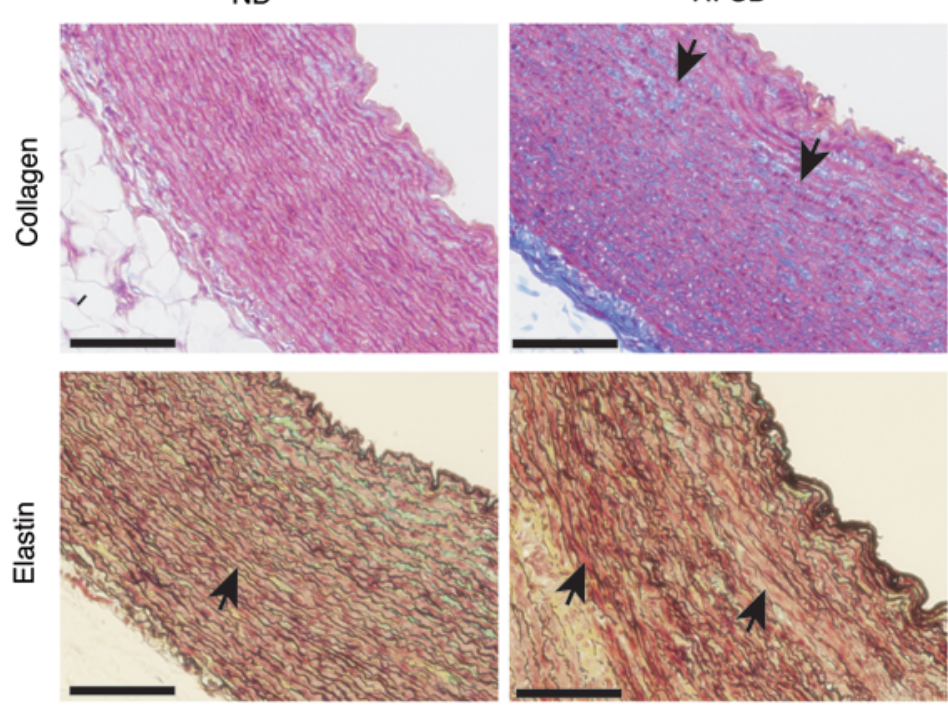

$\mathbf{F}$

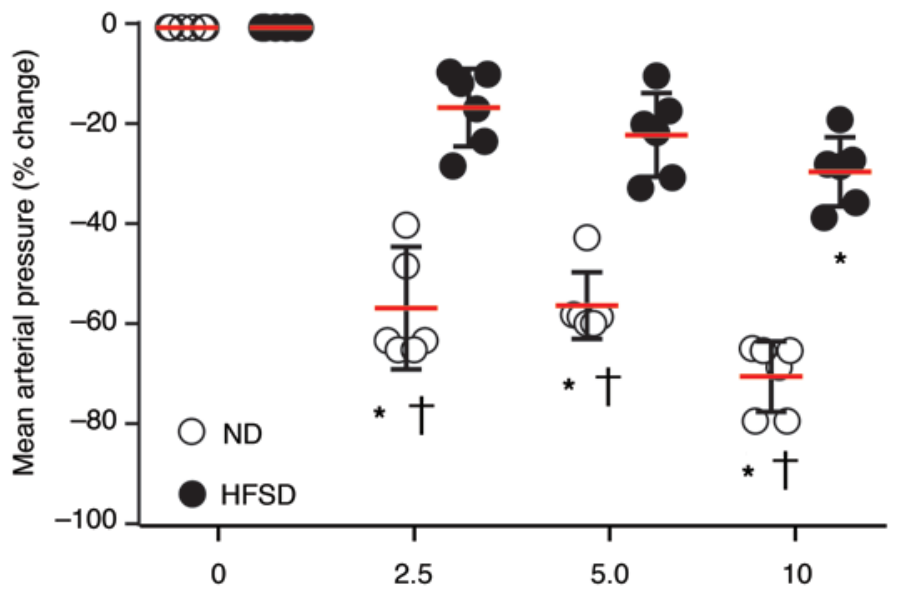

Acetylcholine dose $(\mu \mathrm{g})$

Figure 1. Vascular remodeling and endothelial dysfunction are increased in guinea pigs on a high-fat and high-sucrose diet (HFSD) compared with normal diet (ND) animals. (A) Light microscopy images of representative aorta sections from guinea pigs after 3 months on either ND or HFSD shown in the upper panel were stained with Masson's trichrome (collagen = blue); sections in the lower panel were stained with the Verhoeff's Van Giesen method (elastin fibers = black/brown; collagen fibers $=$ red). Images were obtained at $\times 100$ magnification and show increased visualization of collagen staining and elastin fragmentation in the vascular media. Scale bars: $100 \mu \mathrm{m}$. (B) Percentage area of collagen-positive staining was increased in aortas after 3 months on HFSD (filled circles) compared with ND (open circles) ( $n=10, P=0.0001$ by a 2-tailed $t$ test). (C) Collagen/elastin ratios were significantly increased in ND compared with HFSD ( $n=10, P=0.0001$ by a 2 -tailed $t$ test); both are indicative of remodeling in elastic arteries. Both (D) systolic blood pressure and (E) pulse pressure significantly increased in HFSD (filled circles) compared with ND (open circles) guinea pigs ( $n=12, P=0.0001$ (systolic blood pressure) by a 2-tailed $t$ test) and ( $n=12, P=0.0001$ (pulse pressure) by a 2-tailed $t$ test). (F) Systemic vascular responsiveness (mean arterial blood pressure) in response to acetylcholine doses was significantly lower in HFSD ( $n=12$, closed circles) compared with ND guinea pigs ( $n=12$, open circles), suggesting significantly reduced endothelial NO availability. ND and HFSD guinea pig mean arterial pressure responses following an ANOVA with a multiple comparisons test demonstrated significant differences between groups $\dagger(0,2.5,5.0$, and $10 \mu \mathrm{g}): P=0.9999(0 \mu \mathrm{g}), P=0.0001$ $(2.5 \mu \mathrm{g}), P=0.0001(5.0 \mu \mathrm{g}), P=0.0001(10 \mu \mathrm{g}) .{ }^{*} P=0.0001$ compared with baseline. All data are presented as individual values with the mean \pm SD. Magnification $=$ ocular lens $(\times 10) \times$ objective.
B

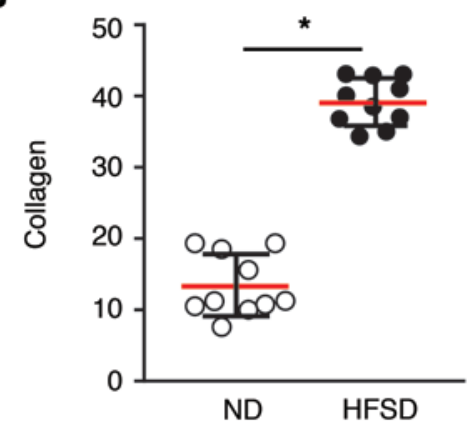

C

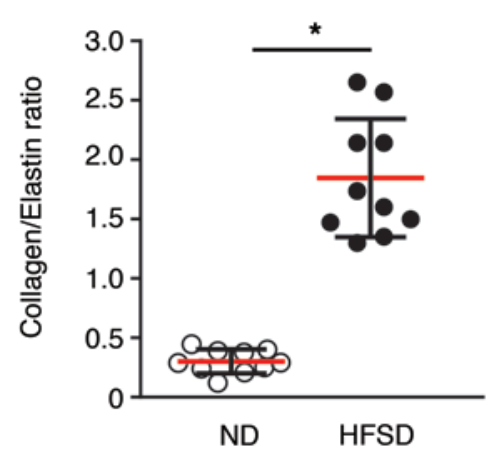

D

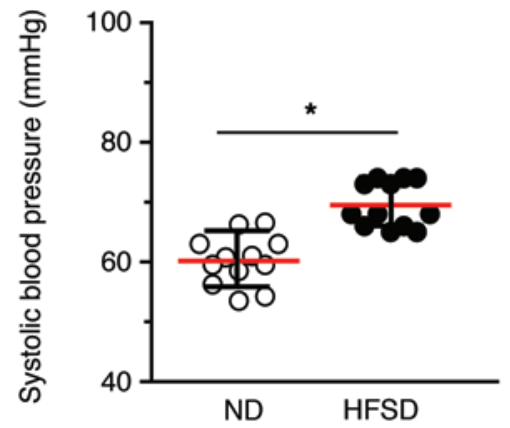

$\mathbf{E}$

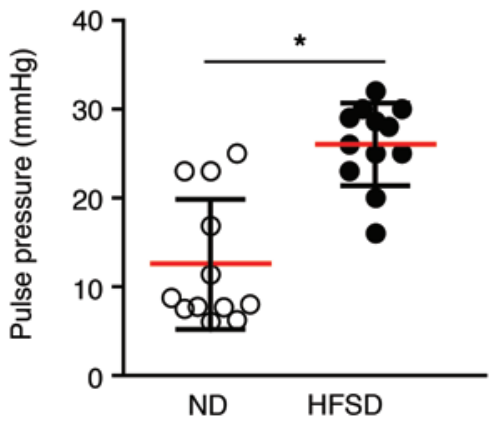


Table 1. RBC storage testing parameters

\begin{tabular}{|c|c|c|}
\hline Analyzed & $\begin{array}{c}\text { Storage day } 1 \text { - Fresh blood } \\
\text { (Mean } \pm \text { SD, } 95 \% \text { CI })(n=4 \text { bags })\end{array}$ & $\begin{array}{l}\text { Storage day } 14 \text { - Stored blood } \\
\text { (Mean } \pm \text { SD, } 95 \% \text { CI) }(n=4 \text { bags) }\end{array}$ \\
\hline 2,3-DPG ( $\mu \mathrm{mol} / \mathrm{g} \mathrm{Hb})$ & $9.39 \pm 1.40(7.16,11.6)$ & $1.65 \pm 0.116(1.47,1.84)^{A}$ \\
\hline ATP $(\mu \mathrm{mol} / \mathrm{g} \mathrm{Hb})$ & $6.53 \pm 0.424(5.86,7.20)$ & $1.30 \pm 0.534(0.415,2.11)^{A}$ \\
\hline Lactate (mmol/l) & $1.68 \pm 0.0500(1.60,1.76)$ & $13.9 \pm 0.287(13.5,14.4)^{A}$ \\
\hline Glucose (g/dl) $)^{B}$ & $123 \pm 30.9(73.3,172)$ & $233 \pm 31.6(183,283)^{A}$ \\
\hline $\mathrm{K}^{+}(\mathrm{mmol} / \mathrm{l})$ & $6.03 \pm 0.0957(5.87,6.18)$ & $8.16 \pm 0.103(7.99,8.32)^{A}$ \\
\hline RBC count $(M / \mu \mathrm{l})$ & $6.13 \pm 0.990(4.43,7.70)$ & $5.32 \pm 0.423(4.64,5.99)$ \\
\hline MCV (fl) & $81.3 \pm 0.419(80.7,82.0)$ & $81.9 \pm 0.351(81.3,82.4)$ \\
\hline НСТ\% & $60.5 \pm 3.1(55.6,65.5)$ & $58.8 \pm 2.87(54.2,63.3)$ \\
\hline WBC count $(K / \mu \mathrm{l})$ & $0.0188 \pm 0.0262(0.0231,0.0606)$ & $0.00700 \pm 0.00294(0.00232,0.00147)$ \\
\hline \multicolumn{3}{|c|}{ 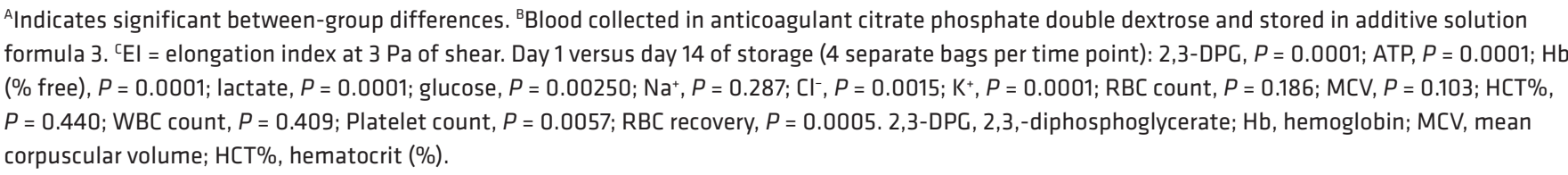 } \\
\hline
\end{tabular}

storage in AS-3 have been performed elsewhere (27) and general comparisons may be useful to the reader. The normal discocyte morphology of guinea pig RBCs under scanning electron microscopy on storage day 1 (Figure 2A) shows pronounced echinocyte/spheroechinocyte forms by day 14 (Figure 2B).

The maximal plasma NTBI concentration after transfusion with 9 units (RBCs administered are referred to here as units rather than mls of total volume exchange transfused) of S-RBCs (260 \pm 80.6 $\mu \mathrm{g} / 1)$ was significantly increased compared with NTBI concentration following transfusion with 9 units of F-RBCs $(50.0 \pm 20.3 \mu \mathrm{g} / 1)$. Administration of apo-Tf $(63.4 \pm 6.77 \mu \mathrm{g} / 1)$, but not $\mathrm{Hp}(322.0 \pm 25.1 \mu \mathrm{g} / 1)$ at the time of 9-unit S-RBC transfusion prevented NTBI accumulation in plasma (Figure 2C). Interestingly, 1- and 3-unit transfusion of S-RBCs generated similar plasma NTBI concentrations to that of 9-unit transfusion (Figure 2C), but did not lead to mortality or tissue injury in the 1- or 3-unit dosing groups. In cotransfused animals, the average maximum plasma concentration of apo-Tf was $20.1 \pm 3.98 \mathrm{mg} / \mathrm{ml}$ and this level declined over 24 hours. Saturation of the infused apo-Tf increased from $0 \%$ to $8.10 \% \pm 3.68 \%$, while NTBI was maintained at pretransfusion levels (Figure 2D). Areas under the NTBI plasma concentration versus time curves $\left(\mathrm{AUC}_{0-24} \mathrm{NTBI}\right)$ show no difference between 1, 3, and 9 units transfused in this model (Figure 2E).

Appearance and accumulation of plasma $\mathrm{Hb}$ was not observed following transfusion of 1 or 3 units of $\mathrm{S}-\mathrm{RBC}$. However, plasma $\mathrm{Hb}$ levels increased to $1.45 \pm 0.45 \mathrm{mg} / \mathrm{ml}$ over the 24 hours after 9 units of S-RBC transfusion (Figure $2 \mathrm{~F})$. Free $\mathrm{Hb}$ in the plasma remained at control levels $(0.012 \pm 0.010 \mathrm{mg} / \mathrm{ml})$ with the administration of $\mathrm{Hp}$ at the time of transfusion (Figure $2 \mathrm{~F}$ ). Plasma levels of infused Hp reached a maximum concentration of $1.97 \pm 0.0810 \mathrm{mg} / \mathrm{dl}$ (Figure $2 \mathrm{G}$ ) and these concentrations exceeded total plasma $\mathrm{Hb}$ concentrations over the course of the study, maintaining $\mathrm{Hb}$ in a $\mathrm{Hb}-\mathrm{Hp}$ complex (Figure 2G, inset). Plasma and $\mathrm{RBC} \mathrm{Hb}$ levels were predominantly in the ferrous $\left(\mathrm{HbFe}^{2+}\right)$ nonoxidized state after transfusion.

$H p$ and apo-Tf administration prevent stored RBC transfusion-related mortality in guinea pigs with preexisting vascular remodeling and endothelial dysfunction. Vascular dysfunction-phenotype HFSD animals $(n=10)$ were dosed with either 9 units of F-RBCs or 1, 3, or 9 units of S-RBC ( $n=10 /$ group). Animals were monitored over the 7 days after transfusion for the onset of humane end-point parameters (labored breathing, shivering with ruffled fur, ataxia with impaired mobility, and inability to reach food and water) and survival. Transfusion with 9 units of S-RBCs led to a significant $(P=0.0005)$ reduction of 7 -day survival $(40 \%, 4$ survivors out of 10) in HFSD guinea pigs compared with ND guinea pigs (Figure 3A). In contrast, transfusion with 9 
A
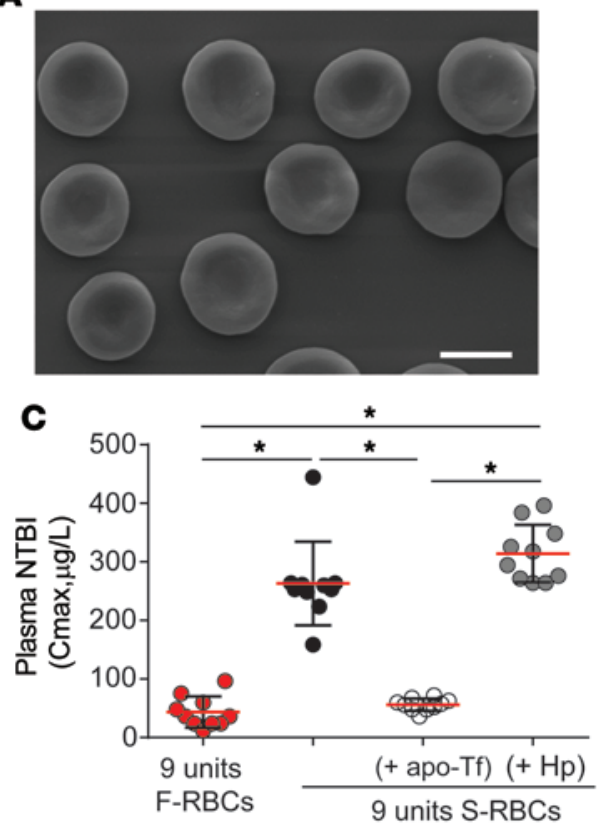

E

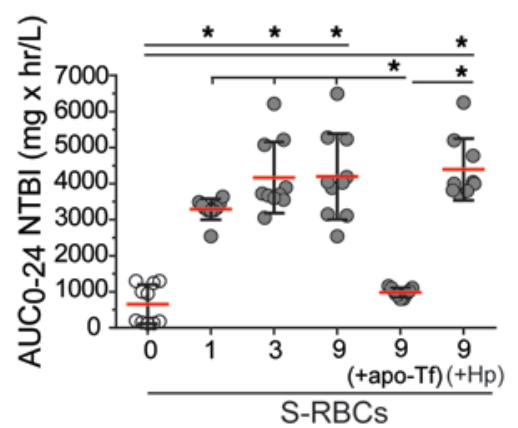

G

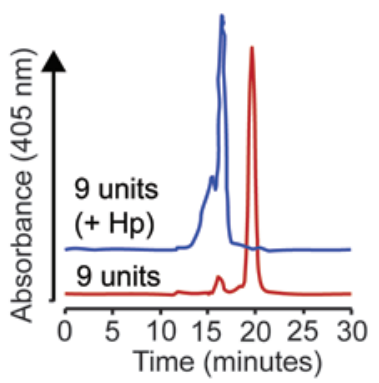

B

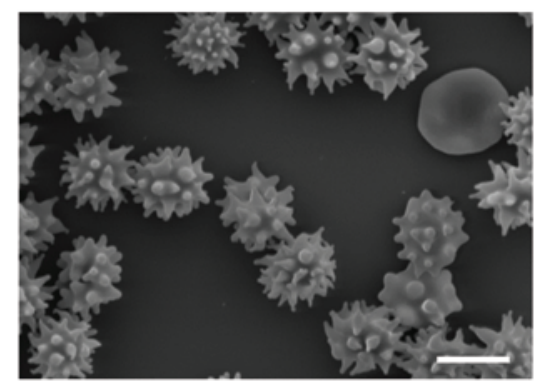

D
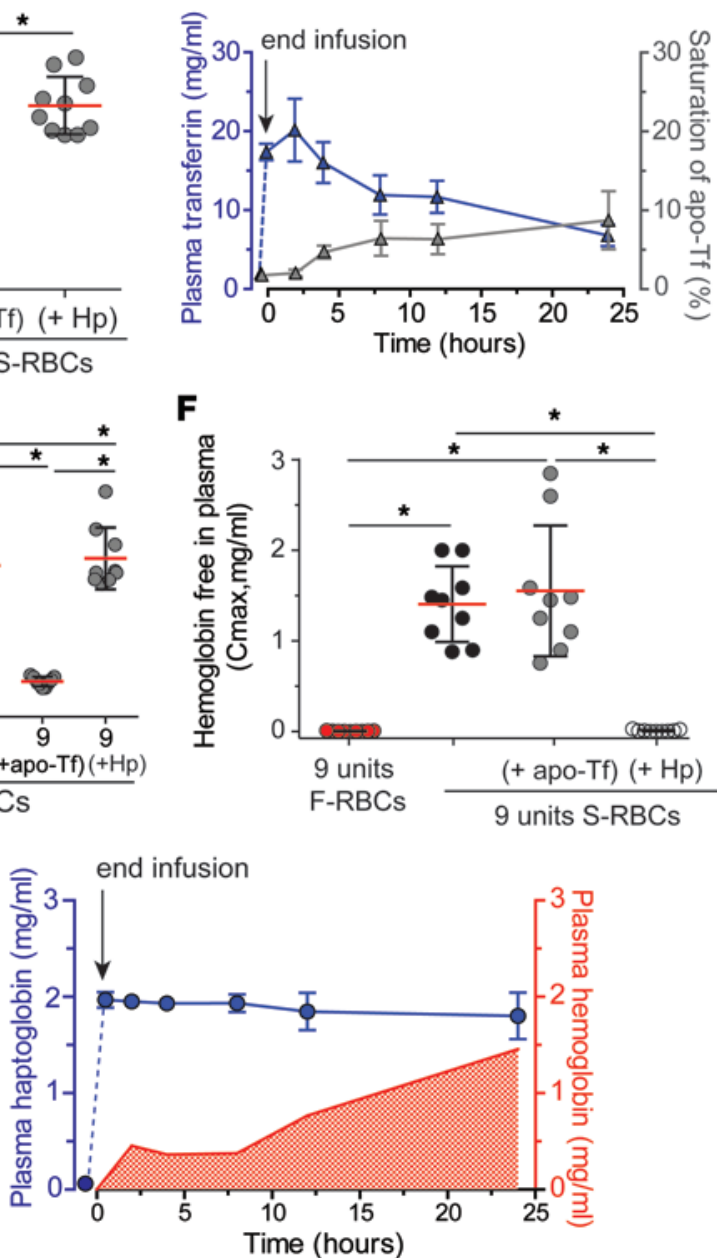

Figure 2. Plasma iron and cell-free hemoglobin (Hb) following fresh red blood cell (F-RBC) and stored red blood cell (S-RBC) transfusion in guinea pigs is prevented with infusion of apo-transferrin (apo-Tf) and haptoglobin (Hp), respectively. ( $\mathbf{A}$ and $\mathbf{B})$ Electron microscopy images of donor guinea pig RBCs on storage day 1 and storage day 14 , respectively. Scale bars: $5 \mu \mathrm{m}$. Original magnification, $\times 5,000$. (C) Maximum plasma non-transferrin-bound iron (NTBI) concentrations (Cmax) were significantly increased following 9-unit transfusion of S-RBCs compared with F-RBCs ( $n=10, P=0.0001)$, coadministration with apo-Tf significantly decreased NTBI ( $n=10, P=0.0001)$, while Hp had no effect on plasma NTBI levels. Significant increases in plasma NTBI were also observed following F-RBC transfusion compared with S-RBC $+\mathrm{Hp}$ $(n=10, P=0.0001)$ and between S-RBC + apo-Tf and S-RBC $+\mathrm{Hp}(n=10, P=0.0001)$. (D) Plasma apo-Tf concentrations $(n=5)$ versus time (blue, left axis) and the saturation of infused apo-Tf ( $n=5$, right gray axis) are shown for qualitative visualization. (E) NTBI plasma concentration versus time areas (AUCs) show no difference between 1,3 , and 9 units of S-RBCs in this model. Transfusion with S-RBCs at 1,3 , and 9 units, and 9 units $+\mathrm{Hp}$ significantly increased plasma NTBI compared with 9 units S-RBCs + apo-Tf ( $n=$ $10, P=0.0001)$. (F) Maximum plasma $\mathrm{Hb}$ concentrations (Cmax) increased compared with 9-unit F-RBC transfusion as follows: 9 units F-RBCs versus 9 units S-RBCs $(n=10, P=0.0140) ; 9$ units F-RBCs versus 9 units S-RBCs + apo-Tf ( $n=$ $10, P=0.0210$ ). While Hp significantly decreased maximum $\mathrm{Hb}$ concentrations compared with 9 units S-RBCs $(n=10, P=0.0151)$ and 9 units S-RBCs + apo-Tf ( $n=10, P=0.0191)$. (G) Plasma $\mathrm{Hp}$ concentrations (blue, left axis) and mean plasma $\mathrm{Hb}$ concentrations $(\mathrm{mg} / \mathrm{ml})$ after transfusion with 9 units S-RBCs (red line/area, right axis) are shown to qualitatively demonstrate that $\mathrm{Hp}$ exceeds $\mathrm{Hb}$ at all time points. Inset is a representative size-exclusion chromatograph showing that all $\mathrm{Hb}$ is bound to $\mathrm{Hp}$ (red trace represents $\mathrm{Hb}$ and blue trace represents $\mathrm{Hb}-\mathrm{Hp}$ in plasma). All statistical comparisons were made using an ANOVA with a multiple comparisons test. All data are presented as individual values with the mean $\pm \mathrm{SD}$.

units of F-RBCs as well as lower doses of S-RBCs (1 and 3 units) had no effect on 7-day survival (Figure 3B). Additionally, we did not observe any deaths after transfusion of 9 units of S-RBCs in healthy ND guinea pigs.

Administration of $\mathrm{Hp}$ or apo-Tf at the time of transfusion rescued HFSD animals receiving 9 units of S-RBC transfusion to $100 \%$ (10 of 10 animals) survival (Figure 3B). In representative animals having expired after 9 units of S-RBC transfusion, pathological changes were associated with large vessel thrombus deposition observed in regions of the aorta (Figure 3C), accentuated vascular injury (elastin fragmentation and collagen deposition) (Figure 3, D and E), renal cortex proximal and distal tubule necrosis, and finally glomerular capillary thrombosis (Figure $3 \mathrm{~F}$ ).

Free iron enhances the oxidation of free $\mathrm{Hb}$, leading to tissue oxidation and injury. Because NTBI was the same at 1, 3, and 9 units of S-RBC transfusion and free $\mathrm{Hb}$ only increased after transfusion of 9 units of S-RBCs, we tested the concept that free iron increases the rate of oxidation of $\mathrm{Hb}$ in vitro and in vivo. We first tested the spectrally derived autoxidation from ferrous $\mathrm{Hb}\left(\mathrm{HbFe}^{2+}\right)$ to ferric $\mathrm{Hb}\left(\mathrm{HbFe}^{3+}\right)$ by adding $50 \mu \mathrm{M}$ iron as 
A Survival proportions

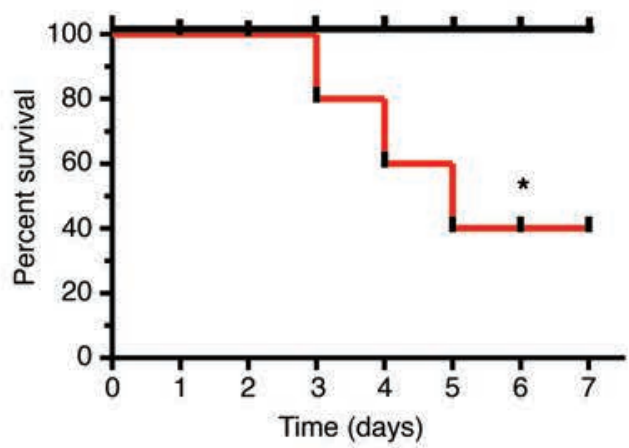

- ND (9 units) S-RBCs - HFSD (9 units) S-BRCS

C

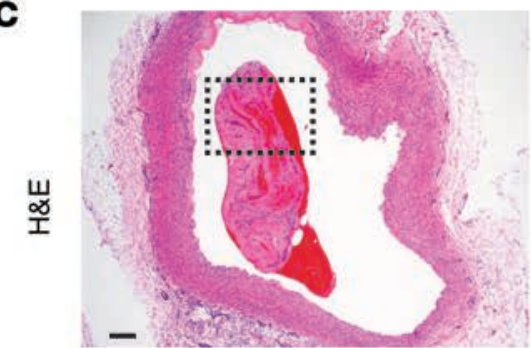

D

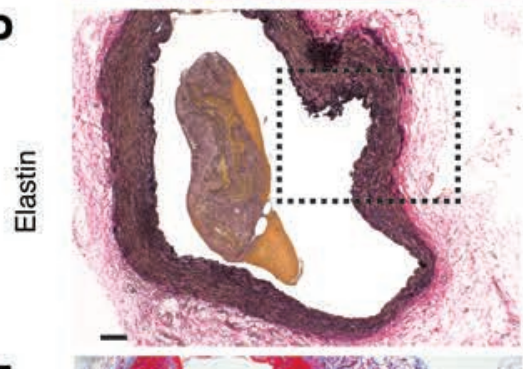

E
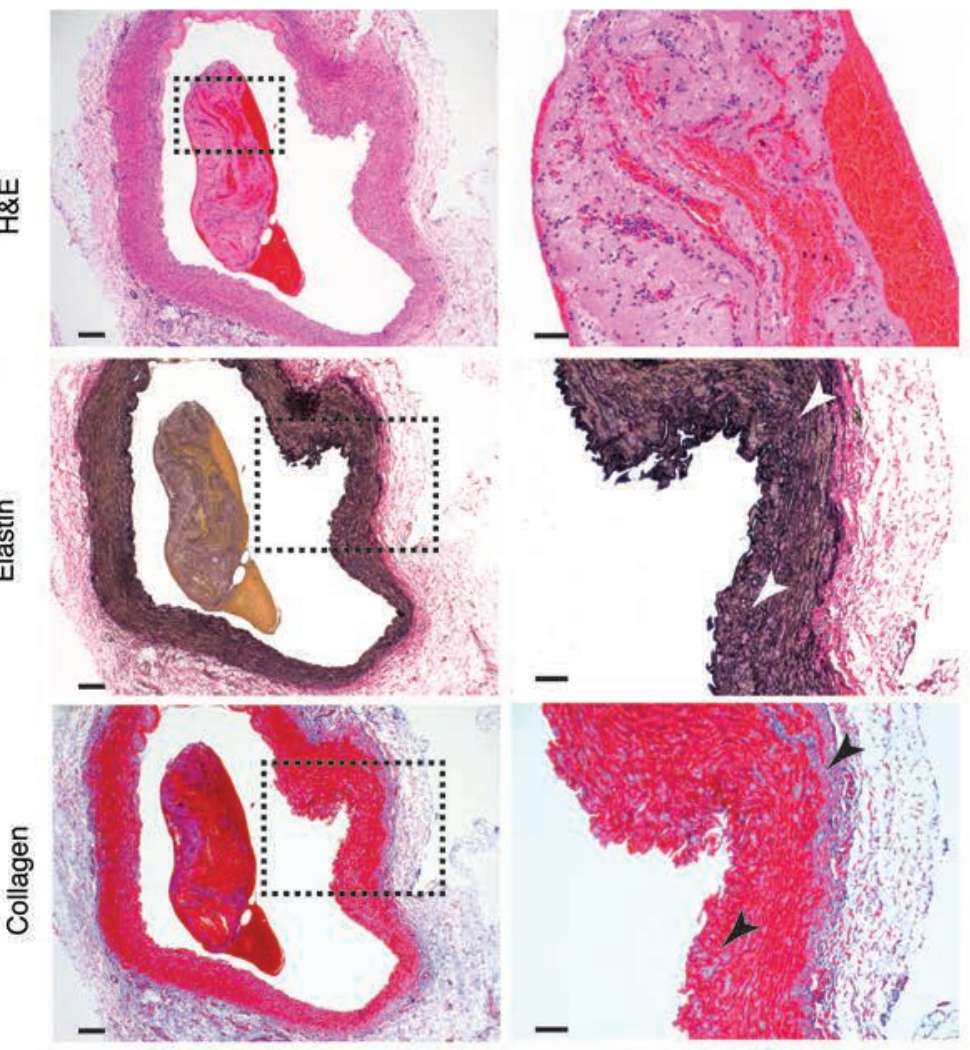

$\mathbf{F}$

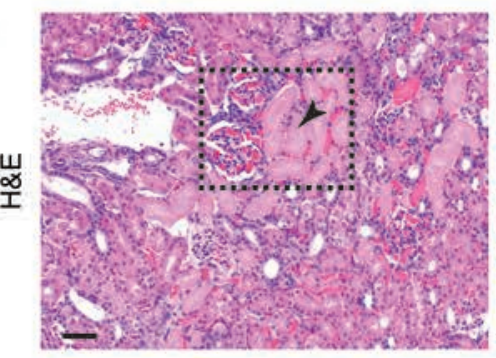

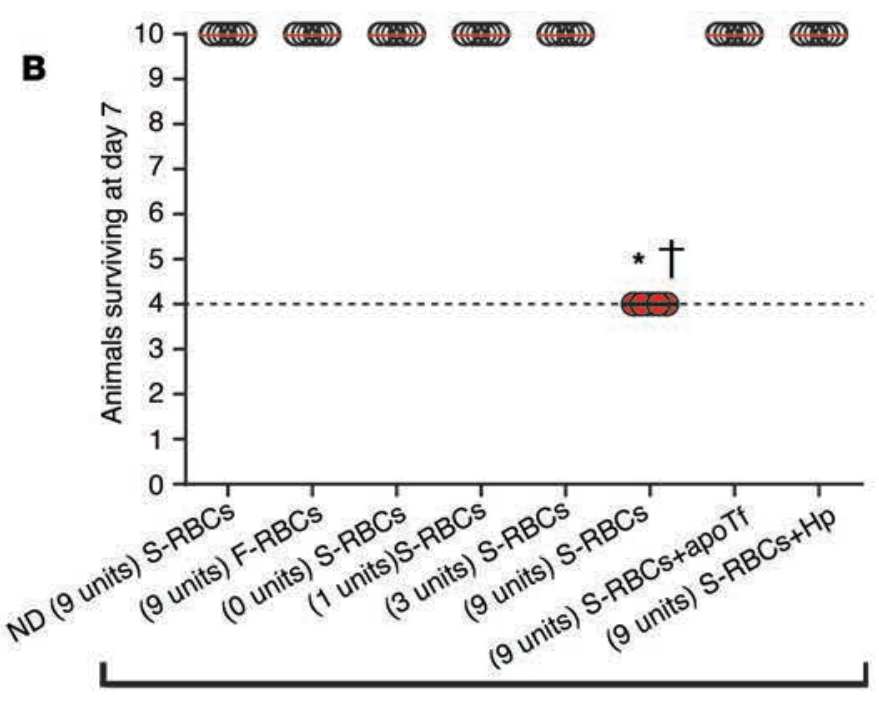

HFSD

Figure 3. Transfusion-related mortality in guinea pigs with preexisting vascular remodeling and endothelial dysfunction is reversed by apo-transferrin (apo-Tf) and haptoglobin (Hp). (A) Nine units S-RBCs significantly increased 7-day mortality in high-fat and high-sucrose diet (HFSD) but not in normal diet (ND) guinea pigs over a 7-day observation period ( $n=10, P=0.0143$, by log-rank [Mantel-Cox]); data are presented as percentage survival. (B) Survival after a 7-day observation period of ND and HFSD guinea pigs after different transfusion regimens with increasing RBC transfusion dose as indicated. Administration of apo-Tf $(300 \mathrm{mg} / \mathrm{kg})$ and $\mathrm{Hp}(300 \mathrm{mg} / \mathrm{kg})$ at the time of transfusion restores survival in the HFSD animals dosed with 9 units of S-RBCs at the highest RBC transfusion dose (apo-Tf; $n=10,{ }^{*} P=0.0001$, ANOVA with a multiple comparisons test) and ( $\mathrm{Hp} ; n=10, \dagger P=0.0001$, ANOVA with a multiple comparisons test). (C) Left panel: H\&E staining of thoracic aorta showing large thrombi in a nonsurviving 9-unit S-RBC-transfused animal. Original magnification, $\times 400$. Scale bar: $200 \mu \mathrm{m}$. Right panel: High magnification of dashed box region. Original magnification, $\times 200$. Scale bar: $50 \mu \mathrm{m}$. (D) Left panel: Verhoeff-Van Geison staining of elastic fibers from the same animal in C. Original magnification, $\times 400$. Scale bar: $200 \mu \mathrm{m}$. Right panel: High magnification of dashed box region showing extensive elastin fragmentation indicated by white arrows. Original magnification, $\times 100$. Scale bar: $50 \mu \mathrm{m}$. (E) Left panel: Masson's trichrome staining of collagen from the same animal in C. Original magnification, $\times \mathbf{4 0 0}$. Scale bar: $200 \mu \mathrm{m}$. Right panel: High magnification of dashed box region showing collagen deposition indicated by solid arrows. Original magnification, $\times 100$. Scale bar: $50 \mu \mathrm{m}$. (F) Left panel: H\&E staining of renal cortical tissue from a separate nonsurviving animal showing acute proximal and distal tubule necrosis (solid arrow). Original magnification, $\times 200$. Scale bar: 50 $\mu \mathrm{m}$. Right panel: High magnification of dashed box region showing loss of renal cortical tubule nuclei (solid arrows) and thrombi occurring in the glomerular capillary structure (white arrow). Original magnification, $\times 600$. Scale bar: 20 $\mu \mathrm{m}$. All images were captured identically with default settings. Magnification $=$ ocular lens $(\times 10) \times$ objective. 
iron sulfate $\left(\mathrm{FeSO}_{4}\right)$ to $100 \mu \mathrm{M} \mathrm{Hb}$ (ferrous, $\mathrm{HbFe}^{2+}$ ) performed in $3(n=3)$ analytical replicates per group at $37^{\circ} \mathrm{C}$. All solutions were monitored by UV-visible spectrometry over a 4-hour period. The addition of iron resulted in an acceleration in the rate of autoxidation of $\mathrm{HbFe}^{2+}$ to $\mathrm{HbFe}^{3+}\left(\mathrm{Hb}\right.$ ox $\left.=7.51 \times 10^{-3} \mu \mathrm{M} / \mathrm{h}\right)$ compared with $\mathrm{Hb} / \mathrm{FeSO}_{4}\left(\mathrm{Hb}\right.$ ox $=1.41 \times 10^{-1} \mu \mathrm{M} / \mathrm{h}$ ) (Figure 4, A and $\left.\mathrm{B}\right)$. The addition of increasing amounts of apo-Tf $(25,50$, and $100 \mu \mathrm{M})$ demonstrated a concentration-dependent decrease in the rate of $\mathrm{Hb}$ oxidation. A maximum reduction of 16 -fold in iron-induced $\mathrm{Hb}$ oxidation was observed after the addition of $100 \mu \mathrm{M}$ apo-Tf $\left(\mathrm{Hb}\right.$ ox $\left.=3.45 \times 10^{-2} \mu \mathrm{M} / \mathrm{h}\right)$ (Figure $4, \mathrm{~A}$ and $\left.\mathrm{B}\right)$.

To understand if this in vitro observation had in vivo significance, tissue oxidation was assessed in formalin-fixed and paraffin-embedded tissue sections from thoracic aorta and kidney following 9-unit S-RBC transfusions ( \pm apo-Tf or Hp). Immunohistochemistry for detecting lipid peroxide-based tissue 4-hydroxynonenal (4-HNE) adducts was performed and shows diffuse anti-4-HNE immunoreactivity in the aorta of representative 9-unit S-RBC-transfused animals (Figure 4C). These data are consistent with our recent observations $(28,29)$. Aortic tissue 4-HNE immunoreactivity observed in the 9-unit S-RBCtransfused group was not observed in animals coadministered apo-Tf or Hp (Figure 4C). Similar observations were made in regions of the renal cortex (Figure 4D).

The effect of localized oxidation on vascular and renal tissue pathology can be visualized by comparing the H\&E-stained thoracic aorta and renal cortex following 9-unit S-RBC transfusions alone and 9-unit S-RBC ( \pm apo-Tf or $\mathrm{Hp}$ ). In the thoracic aorta, clear coagulative necrosis is observed following 9-unit S-RBC transfusion. Iron accumulation in the 9-unit S-RBC-transfused animals is observed in the media and adventitia of aortic vessels (Figure 5A). Apo-Tf and $\mathrm{Hp}$ independently attenuated this effect (Figure 5, A and B). In the renal cortex a similar pattern was observed. Increased renal tubule inflammation and necrosis occurred following 9-unit S-RBC transfusion. However, this was also not observed if apo-Tf or Hp was dosed at the time of transfusion (Figure 5C). The injury marker neutrophil gelatinase-associated lipocalin (NGAL or LCN2) mRNA expression was evaluated as a biological marker to suggest acute renal injury. In the 9-unit S-RBC transfusion group, a significant NGAL basal increase was observed (17.5 \pm 7.85 -fold). Conversely, NGAL expression was significantly attenuated following 9-unit S-RBC transfusion with apo-Tf (7.46 \pm 3.71 , fold induction over baseline) and $\mathrm{Hp}(3.23 \pm 1.61$, fold induction over baseline) administration (Figure 5D).

\section{Discussion}

The present study focused on the dose-dependent mortality associated with the transfusion of older stored RBCs, which can represent as much as $20 \%$ of RBC units awaiting transfusion (1). In the model presented, we observed that the transfusion dose, the storage age of the RBC, and preexisting vascular disease are criti$\mathrm{cal}$ and mutually interactive determinants of posttransfusion mortality. Posttransfusion release of $\mathrm{Hb}$ and NTBI are 2 pathophysiological factors contributing to this end point. Posttransfusion mortality could be prevented when cell-free $\mathrm{Hb}$ or NTBI were neutralized by their binding proteins $\mathrm{Hp}$ or apo-Tf, respectively.

Storage-time-dependent changes of RBCs are characterized by echinocytic shape changes associated with decreased deformability, rapid splenic sequestration, and macrophage clearance (12, 30). Splenic sequestration of transfused RBCs ultimately leads to iron release from macrophages measured as NTBI in plasma and was previously shown to be a mediator of transfusion-related inflammation in animals and possibly increased infection virulence in human patients $(2,31)$. Consistent with these findings, we observed maximum plasma NTBI concentrations in our model within the first 3 hours after S-RBC administration, with levels slowly declining over a 24-hour period. Posttransfusion increases in Tf saturation and subsequent hepcidin elevation appear not to be sufficient to control excess iron in circulation (21). We have therefore investigated if administration of apo-Tf might control NTBI accumulation in this situation. In our studies, infusion of apo-Tf at the time of S-RBC transfusion resulted in a total $\mathrm{Tf}$ plasma concentration of $10 \mathrm{mg} / \mathrm{ml}$ lasting up to 14 hours after infusion, followed by a decline toward baseline. These Tf levels returned NTBI to baseline levels by increasing saturation of infused apo-Tf.

Cell-free $\mathrm{Hb}$ has been considered as another mediator of stored blood transfusion-related adverse events $(13,20)$. Depletion of the vasodilator NO by the dioxygenase reaction, oxidative Hb reactions, and heme release are among the causative mechanisms that have been discussed (13). In our current study, transfusions with 9 units of S-RBCs was associated with moderate, but sustained in vivo intravascular hemolysis, which led to accumulation of cell-free $\mathrm{Hb}$ in plasma. The degree of in vivo hemolysis becomes particularly visual in the $\mathrm{Hp}$-treated animals, in which all released $\mathrm{Hb}$ is captured over time in $\mathrm{Hb}-\mathrm{Hp}$ complexes. No intravascular hemolysis occurred in the 1- and 3-unit transfusion groups, 
A

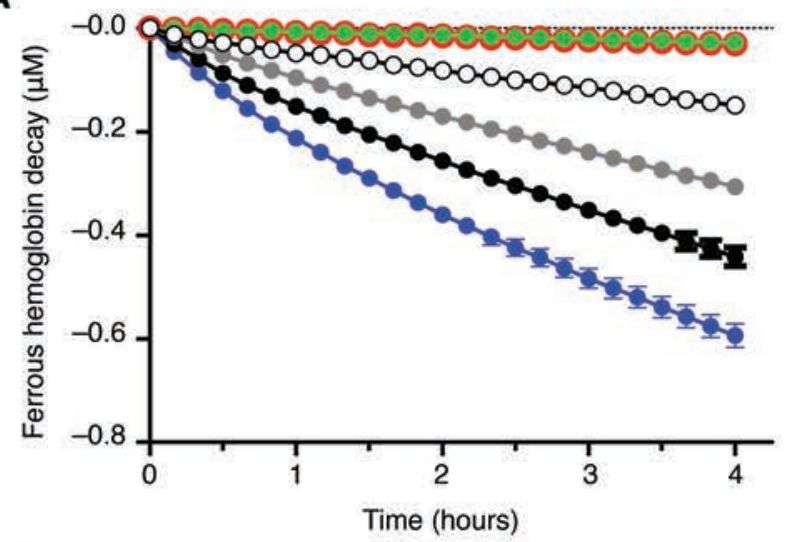

B

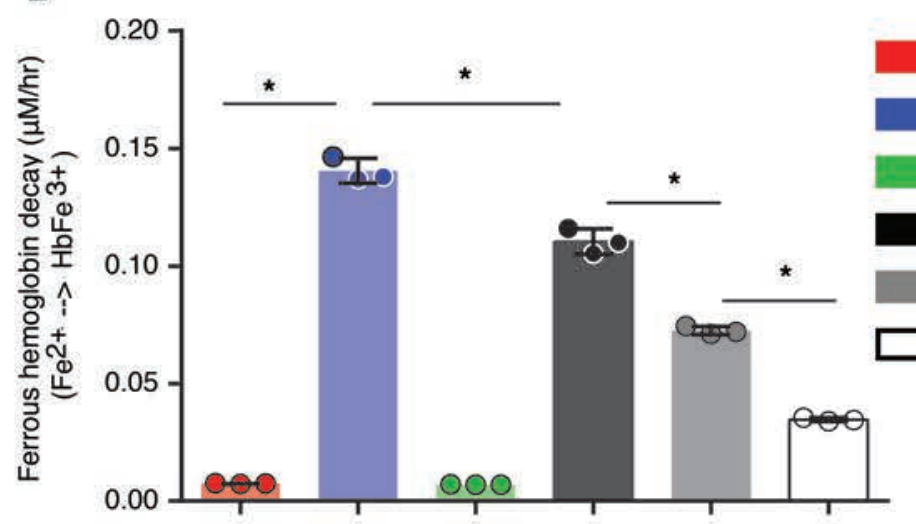

C

Aorta

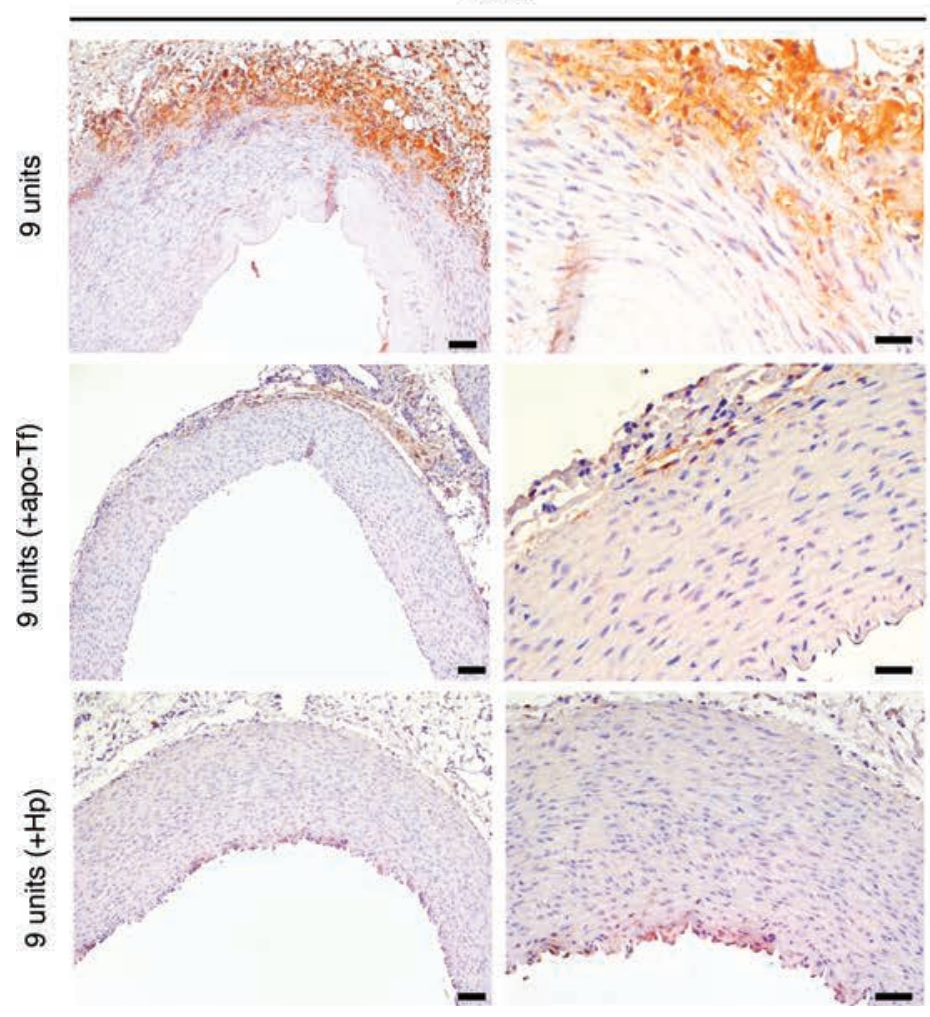

$\mathrm{Hb}(100 \mu \mathrm{M})$

$\mathrm{Hb} / \mathrm{Fe}(50 \mu \mathrm{M})$

$\mathrm{Hb} / \mathrm{apo}-\mathrm{Tf}(100 \mu \mathrm{M})$

$\mathrm{Hb} / / \mathrm{Fe}(50 \mu \mathrm{M}) / \mathrm{apo}-\mathrm{Tf}(25 \mu \mathrm{M})$

$\rightarrow \mathrm{Hb} / \mathrm{Fe}(50 \mu \mathrm{M}) / \mathrm{apo}-\mathrm{Tf}(50 \mu \mathrm{M})$

$\mathrm{Hb} / / \mathrm{Fe}(50 \mu \mathrm{M}) / \mathrm{apo}-\mathrm{Tf}(100 \mu \mathrm{M})$

$\mathrm{Hb}(100 \mu \mathrm{M})$

$\mathrm{Hb} / \mathrm{Fe}(50 \mu \mathrm{M})$

$\mathrm{Hb} / \mathrm{apo}-\mathrm{Tf}(100 \mu \mathrm{M})$

$\mathrm{Hb} / / \mathrm{Fe}(50 \mu \mathrm{M}) / \mathrm{apo}-\mathrm{Tf}(25 \mu \mathrm{M})$

$\mathrm{Hb} / \mathrm{Fe}(50 \mu \mathrm{M}) / \mathrm{apo}-\mathrm{Tf}(50 \mu \mathrm{M})$

$\mathrm{Hb} / / \mathrm{Fe}(50 \mu \mathrm{M}) / \mathrm{apo}-\mathrm{Tf}(100 \mu \mathrm{M})$

Figure 4. Free iron enhances the oxidation of free hemoglobin ( $\mathrm{Hb}$ ) and this biochemical reaction can be attenuated with increasing concentrations of apo-transferrin (apoTf). (A) The UV-visible spectral decay curves are shown for each of the Hb-iron-apo-

Tf mixing groups. (B) An acceleration in autoxidation rates is observed between the following groups: $\mathrm{Hb}$ versus $\mathrm{Hb} / \mathrm{Fe}(n=3, P=0.0001) ; \mathrm{Hb} /$ Fe versus $\mathrm{Hb} / \mathrm{Fe} / \mathrm{apo}-\mathrm{TF}$ (100 $\mu \mathrm{M})(n=3, P=0.001) ; \mathrm{Hb} / \mathrm{Fe} /$ apo-TF $(100 \mu \mathrm{M})$ versus $\mathrm{Hb} /$ Fe/apo-TF $(50 \mu \mathrm{M})(n=3, P$ $=0.0001) ;$ and $\mathrm{Hb} / \mathrm{Fe} / \mathrm{apo}-\mathrm{TF}$ $(50 \mu \mathrm{M})$ versus $\mathrm{Hb} / \mathrm{Fe} / \mathrm{apo}-\mathrm{TF}$ $(25 \mu \mathrm{M})(n=3, P=0.0001)$. All statistical comparisons were made using an ANOVA with a multiple comparisons test. All data are presented as individual values with the mean \pm SD. (C) 4-Hydroxynonenal immunochemistry of thoracic aorta tissue sections showing brown/orange immunoreactivity on adventitia and media. Left: Original magnification, $\times 100$. Scale bars: $50 \mu \mathrm{m}$. Right: Original magnification, $\times 400$. Scale bars: $20 \mu \mathrm{m}$. (D) 4-Hydroxynonenal immunochemistry of non-necrotic renal cortical tissue sections showing brown/orange immunoreactivity in the renal tubules. Original magnification, $\times 600$. Scale bars: $50 \mu \mathrm{m}$. All images were captured identically in high dynamic range (HDR) mode with default settings. Magnification = ocular lens $(\times 10) \times$ objective. 


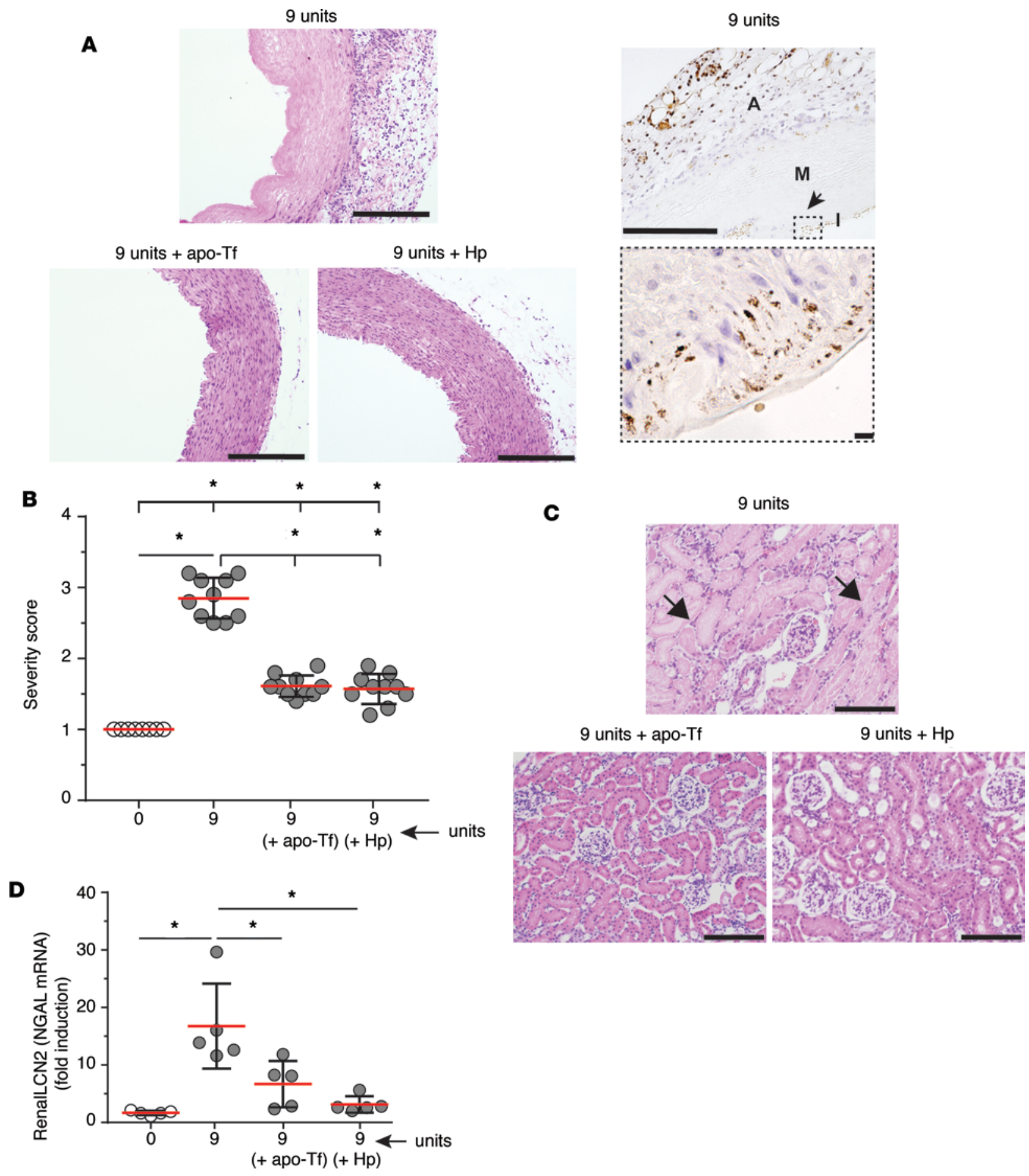

Figure 5. Systemic vascular and renal tissue injury following 9-unit stored red blood cell (S-RBC) transfusion following coadministration of apo-transferrin (apo-Tf) or haptoglobin (Hp). (A) H\&E staining of thoracic aorta tissue of guinea pigs transfused with 9 units S-RBCs and stained for non-heme iron. Left: Original magnification, $\times 100$. Scale bars: $500 \mu \mathrm{m}$. Right: The arrow (top image) represents the region of the high-magnification image (bottom) showing iron deposition within the vascular wall. Original magnification, $\times 100$ (top) and $\times 960$ (bottom). Scale bars, $500 \mu \mathrm{m}$ (top) and $50 \mu \mathrm{m}$ (bottom). A, adventitia; M, media; I, intima. The effect of apo-Tf and Hp infusions are shown (magnification, $\times 100$; scale bar, $500 \mu \mathrm{m}$ ). (B) Aortic injury severity score for each transfusion dose group ( $n=10$ animals). ANOVA with a multiple comparisons test was performed and results are as follows. Comparison with sham control ( 0 units): 0 units versus 9 units S-RBCs ( $n=10, P=0.0001)$. Comparison with 9 units S-RBCs: 9 units S-RBCs versus 9 units S-RBCs + apo-Tf $(n=10, P=0.0001) ; 9$ units S-RBCs versus 9 units S-RBCs $+\mathrm{Hp}(n=10, P=0.0001)$. (C) Light microscopy images of renal cortical tissue from 9-unit S-RBC-transfused stained with H\&E. Original magnification, $\times 200$. Scale bars: $200 \mu \mathrm{m}$. The solid arrows show necrotic tubules with absence of nuclei, lacking intact lumen. The effect of apo-Tf and Hp dosing are shown. (D) Renal LCN2 (NCAL) mRNA expression in kidney tissues is presented as fold induction relative to the expression in control animals. Comparison with sham control ( 0 units): 0 units versus 9 units S-RBCs $(n=5, P=0.0002)$. Comparison between 9 units S-RBCs: 9 units S-RBCs versus 9 units S-RBCs + apo-Tf ( $n$ $=5, P=0.005) ; 9$ units S-RBCs versus 9 units S-RBCs $+\mathrm{Hp}(n=5, P=0.0008)$. All data are presented as individual values with the mean \pm SD. ${ }^{*}$ Indicates significant between-group differences. Magnification $=$ ocular lens $(\times 10) \times$ objective. 
suggesting that cell-free $\mathrm{Hb}$ release occurs only at high transfusion volumes. Presumably, the splenic sequestration capacity for storage-damaged RBCs becomes saturated under these conditions favoring intravascular hemolysis.

Mechanisms similar to those discussed above may explain the data from a retrospective analysis of patients with cardiovascular disease receiving larger volume blood transfusion, which suggested worse outcome in terms of cardiovascular complications and mortality in patients receiving older-storage blood (4). Our experimental data are also consistent with prospective studies where lower volume transfusions of 3 to 4 units of blood showed no mortality difference irrespective of the relative RBC storage period, reinforcing the critical impact of dose-dependent RBC adverse outcomes (7). The vulnerability of mice $(24,25,32)$, lambs (15), and patients (26) with endothelial dysfunction to the pathophysiological effects of $\mathrm{Hb}$ and RBC transfusion has been established by Zapol and colleagues. This group has also convincingly demonstrated that maximally stored RBCs (42-day equivalent) caused a poor resuscitation response in a mouse model of shock $(24,25)$ and a significant increase in pulmonary arterial pressure in obese volunteers (26).

Given the large accumulation of data on the transfusion of stored blood in animals and humans, it is likely novel that 2 potentially toxic mediators (iron and $\mathrm{Hb}$ ) that arise from $\mathrm{RBC}$ breakdown could interact to enhance mortality in an animal model that is particularly susceptible to enhanced oxidative stress. Our preclinical data support that transfusion of stored RBCs increases mortality in combination with vascular disease at maximal $\mathrm{RBC}$ transfusion doses. The concomitant presence of iron and $\mathrm{Hb}$ is critical for the onset of tissue oxidation and injury associated with death in this model. In other conditions, such as sickle cell disease (SCD), where hemolysis-driven NO depletion leads to tissue methemoglobin accumulation, vascular injury, cardiac remodeling, and pulmonary hypertension, it is not typically discussed that interaction between $\mathrm{Hb}$ components may contribute toward alterations in the redox balance within tissue (33); however, the data presented here may suggest novel contributors toward vascular complication in other hemolytic disease states. Outcomes can be reversed by apo-Tf and $\mathrm{Hp}$ such that removal of iron or $\mathrm{Hb}$ negates the interactive toxicity. Therefore, our data suggest that increases in plasma NTBI and Hb resulting from stored-blood transfusion contribute to vascular disease-associated mortality though iron-enhanced $\mathrm{Hb}$ oxidation and subsequent tissue injury.

\section{Methods}

\section{Materials}

Purified human multimeric apo-Tf and Hp were obtained from CSL Behring at concentrations of $25 \mathrm{mg} /$ $\mathrm{ml}$ and $100 \mathrm{mg} / \mathrm{ml}$, respectively. An ND (TD.2040) containing complex carbohydrate (oats-wheat-alfalfa, $57 \%$ calories), fat (mono/poly unsaturated fat, $12 \%$ ), protein (soy isolate, $31 \%$ calories), and HFSD (TD.110484, atherogenic guinea pig diet) containing simple carbohydrate (sucrose, $35 \%$ calories), complex carbohydrate (oats-wheat-alfalfa, $9 \%$ calories), fat (cocoa butter, $38 \%$ calories with $0.25 \%$ cholesterol), and protein (soy isolate, $18 \%$ calories) were prepared by Teklad custom research diets.

\section{Antibodies}

Rabbit polyclonal anti-4-HNE was purchased from Alpha Diagnostic (catalog HNE 12-C).

\section{Animal studies}

All animal studies were approved by the FDA/CBER Institutional Animal Care and Use Committee, with all experimental procedures performed in adherence to the NIH guidelines on the use of experimental animals. Male Hartley guinea pigs weighing 300-400 g were obtained from Charles Rivers Laboratories and acclimated upon arrival at the FDA/CBER animal care facility (Bethesda, MD and The Federal Research facility at White Oak, Silver Spring, MD). Experiments in normal animals were conducted following 12 weeks on ND, while experiments in vascular diseased animals were conducted following 12 weeks on HFSD as previously described (34). Animals allocated to study groups were dosed 12 hours prior to surgery with enrofloxacin $(10 \mathrm{mg} / \mathrm{kg})$ by the subcutaneous route. On days of surgery, guinea pigs were dosed subcutaneously with ketoprofen $(5 \mathrm{mg} / \mathrm{kg}$ ) for pain management after recovery and then anesthetized via the intraperitoneal route with a cocktail of ketamine $\mathrm{HCl}(100 \mathrm{mg} / \mathrm{kg})$ and xylazine $\mathrm{HCl}(5 \mathrm{mg} / \mathrm{kg})(\mathrm{Phoenix}$ Scientific Inc.). Sterilized PE50 tubing catheters were placed in the left external jugular vein, right carotid artery, and left femoral artery. 


\section{Blood collection and preparation for storage and transfusion}

Using aseptic technique, allogeneic blood was collected from ketamine/xylazine-anesthetized donor guinea pigs via a left carotid artery catheter attached to CP2D-filled syringes (Pall Corporation). Collections were pooled and leukoreduced using a neonatal high-efficiency leukocyte reduction filter and storage bag (Purecell Neo, Pall Corporation). Blood was centrifuged at $805 \mathrm{~g}$ for 20 minutes and plasma was removed, and packed RBCs were supplemented with AS-3 (Pall Corporation). All procedures were conducted under sterile conditions. The final preservative concentration was equal to $14 \%$, and storage was maintained in bags at $4^{\circ} \mathrm{C}$ over a 14 -day period. F-RBCs were processed the same way, but used within 1 day of collection.

\section{Transfusion protocol}

Male Hartley guinea pigs on HFSD ( $n=10$ per group) for 3 months received $0,1,3$, or 9 units of S-RBCs in donor plasma (hematocrit 40\%). This is based on normal hematocrit values of $37.3 \% \pm 0.82 \%(n=20$ animals) in male Hartley guinea pigs housed in our animal facility. Volumes were administered as 1.5 , 4.5 , and $13.5 \mathrm{ml}$ by adding donor guinea pig plasma $(0.5,1.5$, and $4.5 \mathrm{ml})$ to $60 \%$ hematocrit $\mathrm{F}$-RBCs or S-RBCs. RBCs administered are referred to here as units rather than mls of total volume exchange transfused. Administration was carried out by sequential exchange transfusion through the jugular and carotid catheters by withdrawing blood at a rate of $1 \mathrm{ml} / \mathrm{min}$ (carotid catheter) and replacing RBCs/plasma at a rate of $1 \mathrm{ml} / \mathrm{min}$ (jugular catheter). An additional 2 groups received 9 units of RBCs in plasma and a concomitant administration of $\mathrm{Hp}$ or apo-Tf $(300 \mathrm{mg} / \mathrm{kg}, n=10)$ given as a 3 -ml volume over 5 minutes. Control groups included guinea pigs receiving ND. All animals were monitored for 7 days after transfusion. Moribund animals were euthanized and classified as nonsurvivors. Remaining animals were euthanized at 7 days after transfusion and classified as survivors.

\section{RBC storage parameters}

RBCs were analyzed on day 1 ( $n=4$ bags) and day 14 of storage ( $n=4$ bags). RBCs were measured using an Abaxis Vetscan VS² (Abbott) with diagnostic-specific rotors for electrolytes, glucose, and lactate. RBC count, mean corpuscular volume, hematocrit, WBC count, and platelet counts were measured using a CELL-DYN hematology analyzer (Abbott). RBC 2,3-diphosphoglycerate was analyzed using a spectrophotometric assay performed according to the manufacturer's instructions (Roche Diagnostics). RBC ATP was analyzed using a luminescence assay performed according to the manufacturer's instructions (Abcam). RBCs were labeled with 5-chloromethylfluorescein diacetate (CellTracker Green CMFDA dye, Life Technologies) according to the manufacturer's protocol. Labeled RBCs $(2.5 \mathrm{ml})$ were administered to male Hartley guinea pigs and blood was sampled 10 minutes, 1 hour, 2 hours, 4 hours, and 24 hours thereafter. Percentage recovery was determined by dividing labeled cells remaining in circulation at 24 hours by labeled cells in the circulation 10 minutes following labeled RBC administration $(\times 100)$.

\section{RBC morphological evaluation}

RBCs were fixed (1\% glutaraldehyde in $0.1 \mathrm{M}$ phosphate buffer) and postfixed with $1 \%$ osmium tetroxide for 1 hour at room temperature. After rinsing in distilled water 3 times, samples were dehydrated in a series of ethanol concentrations (50\%, 70\%, and 95\%) for 10 minutes each, followed by three 10-minute incubations in $100 \%$ ethanol. The samples were then transferred to a pressure chamber for critical point drying (Leica EM CPD300) and gold coated with a high-resolution 150T S sputter coater (EMS). Morphology was examined by scanning electron microscopy (JEOL JSM-6390LV). RBC deformability was measured using ektacytometry (RheoScan-AnD 300, Rheomeditech) at the same time points. The elongation index (EI) was evaluated from 0 to 20 pascals $(\mathrm{Pa})$ and compared at $3 \mathrm{~Pa}$, which is relevant to shear stress experienced by circulating RBCs.

\section{NTBI and Hb measurements in plasma}

NTBI measurement in plasma. Direct colorimetric NTBI detection of plasma $(n=10)$ was performed. Plasma samples were thawed on ice, $800 \mathrm{mM}$ nitrilotriacetic acid (NTA, Sigma-Aldrich) solution (pH 7.0) was added, and samples were incubated for 30 minutes. The solution was then ultrafiltered using $10-\mathrm{kDa}$ microfilters (Merck Millipore). $\mathrm{HCl}$ (1N, Thermo Fisher Scientific) and trichloracetic acid (TCA, Sigma-Aldrich) 10\% in equal volumes were added to the ultrafiltrate. Ascorbic acid $(5 \mathrm{mg} / \mathrm{ml})$ and chromogen solution $(0.508 \mathrm{mmol} / 1$ FerroZine $[\mathrm{HACH}], 1.5 \mathrm{~mol} / 1$ sodium acetate, and $0.1 \%$ or $1.5 \%(\mathrm{v} / \mathrm{v})$ thioglycolic acid in high-purity water) were added. After incubation, absorbance was measured at $562 \mathrm{~nm}$ using a microplate reader (BioTek Synergy). 
Plasma $\mathrm{Hb}$ analysis. Plasma from baseline samples $(n=10)$ of each animal was used to correct for background interference and turbidity and measured using a Hewlett-Packard HP-8453 rapid-scanning diode array spectrophotometer (Agilent Technologies). Ferrous $\left(\mathrm{HbFe}^{2+}\right) \mathrm{Hb}$ (oxy/deoxy), ferric $\mathrm{Hb}\left(\mathrm{HbFe}^{3+}\right)$, and hemichrome were determined using multicomponent analysis based on the extinction coefficients for each species, and total heme was calculated by adding these values and converting heme concentration to total $\mathrm{Hb}$. To confirm hemolysis and subsequent $\mathrm{Hb}$ scavenging by $\mathrm{Hp}$, we analyzed representative samples of each time point in the 9-ml and 9-ml plus Hp group using size-exclusion chromatography as follows. Plasma samples were separated on an analytical BioSep-SEC-S3000 $(600 \times 7.5 \mathrm{~mm})$ column (Phenomenex) with $50 \mathrm{mM}$ potassium phosphate, $\mathrm{pH} 7.4$, as the mobile phase monitored at $\lambda=280 \mathrm{~nm}$ and $\lambda=405 \mathrm{~nm}$. $\mathrm{Hb}$ bound to $\mathrm{Hp}$ and unbound in plasma was determined by dividing the $\mathrm{Hb}$ peak area and the $\mathrm{Hb}-\mathrm{Hp}$ peak areas by additive areas under the $\mathrm{Hb}-\mathrm{Hp}$ chromatographic peak (12- to 17-minute elution) and the $\mathrm{Hb}$ chromatographic peak (21-minute elution) at $\lambda=405 \mathrm{~nm}$.

Tissue pathology (H\&E, collagen, and elastin)

Kidneys and thoracic aortas were fixed in $10 \%$ formalin for 24 hours and then stored in $100 \%$ isopropanol, embedded in paraffin, and 5 - $\mu \mathrm{m}$ sections were prepared.

$H \& E$. Slides were stained with alum hematoxylin, rinsed with deionized water, differentiated with $0.3 \%$ acid alcohol, and stained with eosin for 2 minutes.

Masson's trichrome staining (collagen). Tissue was refixed in Bouin's solution, washed in deionized water, stained in Weigert's iron hematoxylin, Biebrich scarlet-acid fuchsin solution, and finally phosphomolybdic-phosphotungstic acid for differentiation. After dehydrating in graded ethanol and SafeClear (Thermo Fisher Scientific), slides were mounted in Permount (Thermo Fisher Scientific) and coverslipped. Images were obtained using an Olympus IX71 inverted microscope (Olympus America Inc.).

Verhoeff's Van Geisen staining (elastin fibers). Tissue slides were immersed in Verhoeff's hematoxylin for 30 minutes, differentiated in $2 \%$ ferric chloride, washed with deionized water, and counterstained with Van Gieson's stain for 5 minutes, dehydrated, and coverslipped. All images were captured identically in high dynamic range (HDR) mode with default settings.

\section{Morphometric/image analysis}

When taking images representing 1 dataset, camera or microscope settings remained unchanged to ensure consistency. Images of thoracic aorta were taken at $\times 10$ magnification and 1,200 $\times 1,600$ pixel resolution; the whole tissue section was captured in 10 nonoverlapping pictures. Images of slides stained by Masson's trichrome (collagen) and Verhoeff's Van Geison (elastin) methods were obtained at $\times 100$ and $\times 200$ magnification. Aortic tissue sections were prepared from $n=10$ animals. Images were captured at $\times 200$ magnification and a resolution of $1,200 \times 1,600$ pixels. When taking images to assess collagen and elastin, the camera and microscope settings remained unchanged to ensure image consistency. Analysis was performed on 20 nonoverlapping images per slide ( $n=5$ slides $/ 10$ animals). The area of Masson's trichrome (collagen, blue-stained tissue) and Verhoeff's Van Geison (elastin, black-stained tissue) was obtained by batch analysis using ImageJ software (NIH) and divided by the total area (stained + nonstained), and then multiplied by 100 to give the percentage staining per image. Data are represented as mean $\pm \mathrm{SD}$ percentage stained area.

\section{4-HNE immunohistochemistry}

Paraffin-embedded 5- $\mu \mathrm{m}$ sections were dewaxed, rehydrated, and heat treated in a microwave oven for 15 minutes in $10 \mathrm{mM}$ sodium citrate buffer, $\mathrm{pH}$ 6.0. After cooling for 30 minutes at room temperature, slides were washed in phosphate-buffered saline with $0.05 \%$ Tween 20 (PBST) and incubated with Bloxall (Vector Laboratories) for 15 minutes at room temperature to inhibit endogenous peroxidases. After a brief wash, sections were blocked in $2.5 \%$ horse serum for 30 minutes at room temperature and then incubated overnight at $4^{\circ} \mathrm{C}$ with antibodies against 4-HNE (Alpha Diagnostic) in PBST containing $2.5 \%$ horse serum. Signal was developed using polymeric peroxidase-conjugated secondary antibodies (Vector ImmPRESS Kit) and DAB (SigmaFast DAB, Sigma-Aldrich). Slides were then dehydrated in graded ethanol and SafeClear, and mounted using Permount. All images were acquired using an Olympus IX71 inverted microscope equipped with an Olympus DP70 digital camera. All images were captured identically in HDR mode with default settings. 


\section{Blood pressure measurements and Ach test for vascular responsiveness}

Basal blood pressures were monitored for 60 minutes in ND and HFSD animals ( $n=12$ per group). Ach was administered at 5 doses ranging from $1.25 \mu \mathrm{g}$ to $10 \mu \mathrm{g}$ through a venous jugular catheter in conscious guinea pigs; blood pressure was simultaneously monitored from a carotid artery catheter coupled to a Gould Statham pressure transducer (Beckton Dickenson Critical Care) with data acquired using a Biopac MP150 data acquisition system (Biopac systems Inc.). Injection volume was normalized to $0.25 \mathrm{ml}$ for each dose and administered over 5 minutes. The nadir mean arterial blood pressure was allowed to return to baseline prior to administration of each dose.

\section{Measurement of Hb oxidation in the presence of iron and apo-Tf}

Autoxidation experiments were carried out as described previously (35). $\mathrm{Hb}(100 \mu \mathrm{M})$ was incubated in phosphate buffered saline ( $\mathrm{pH} 7.4)$, and visible spectra $(400-700 \mathrm{~nm})$ were recorded at every 10 minutes for a 4-hour incubation at $37^{\circ} \mathrm{C}$. Another set of autoxidation experiments were performed in the presence of ferrous iron $\left(50 \mu \mathrm{M}\right.$ as $\left.\mathrm{FeSO}_{4}\right)$ alone or with Tf. The spectral changes in oxidation state over time were monitored in a Cary 60 temperature-controlled photodiode array spectrophotometer (Agilent). The absorbance changes at $576 \mathrm{~nm}$ due to the spontaneous oxidation of oxyHb were plotted over time and analyzed by nonlinear least-square curve fitting to single- or double-exponential equations using Microsoft Excel to obtain oxidation rates. Mean oxidation rates with SDs were obtained by averaging data from at least 3 different sets of experiments.

\section{Statistics}

Data are represented as mean \pm SD for parametric data. A Shapiro-Wilk test was performed to determine normal distribution of data. For parametric data, planned comparisons between RBC doses, Hp, and apoTf treatment ( $n=10$ /group or as indicated in figure legends for individual assays) were analyzed using a 1-way ANOVA with Bonferroni's correction for multiple comparisons. Group comparison for basal diet changes were analyzed using an unpaired Student's $t$ test. Blood storage parameters analysis was performed using a paired Student's $t$ test and represented as mean \pm SD with the $95 \%$ CI. Survival proportions for transfused ND and HFSD guinea pigs ( $n=10$ /group) were analyzed using a log-rank (Mantel-Cox) test. All statistical analysis was performed using GraphPad Prism version 6.1.

\section{Author contributions}

JHB designed the experimental work, performed research, analyzed data, and reviewed the manuscript. AY, YG, and RG performed research, analyzed data, and reviewed the manuscript. DRS designed the experimental work, wrote the final version of the paper, and reviewed the manuscript. DJS and PWB designed the experimental work, performed research, analyzed data, wrote the final version of the manuscript, and reviewed the manuscript.

\section{Acknowledgments}

The studies were supported by FDA funding to P.W.B. and J.H.B. Research funding for R.G. was supported by the University Hospital of Zurich. D.J.S. received funding for the study from the Swiss National Science Foundation (31003A/138500) and the Swiss Federal Commission for Technology and Innovation. The authors thank Nathan Brinkman (CSL Behring) for preparing and providing human plasma-derived Hp and apo-Tf for the experiments performed in this report.

Address correspondence to: Paul W. Buehler, Laboratory of Biochemistry and Vascular Biology, Center for Biologics Evaluation and Research, FDA, 10903 New Hampshire Avenue, Silver Spring, Maryland 20993, USA. Phone: 240.402.9411; E-mail: paul.buehler@fda.hhs.gov.

1. Glynn SA, Klein HG, Ness PM. The red blood cell storage lesion: the end of the beginning. Transfusion. 2016;56(6):1462-1468.

2. Remy KE, et al. Transfusion of recently donated (fresh) red blood cells (RBCs) does not improve survival in comparison with current practice, while safety of the oldest stored units is yet to be established: a meta-analysis. Vox Sang. 2016;111(1):43-54

3. Flegel WA, Natanson C, Klein HG. Does prolonged storage of red blood cells cause harm? Br J Haematol. 2014;165(1):3-16.

4. Koch CG, et al. Morbidity and mortality risk associated with red blood cell and blood-component transfusion in isolated coronary artery bypass grafting. Crit Care Med. 2006;34(6):1608-1616. 
5. Koch CG, et al. Duration of red-cell storage and complications after cardiac surgery. N Engl J Med. 2008;358(12):1229-1239. 6. Lacroix J, et al. Age of transfused blood in critically ill adults. N Engl J Med. 2015;372(15):1410-1418.

7. Steiner ME, et al. Effects of red-cell storage duration on patients undergoing cardiac surgery. N Engl J Med. 2015;372(15):1419-1429.

8. Wang D, Sun J, Solomon SB, Klein HG, Natanson C. Transfusion of older stored blood and risk of death: a meta-analysis. Transfusion. 2012;52(6):1184-1195.

9. Edgren G, et al. Duration of red blood cell storage and survival of transfused patients (CME). Transfusion. 2010;50(6):1185-1195.

10. Fergusson DA, et al. Effect of fresh red blood cell transfusions on clinical outcomes in premature, very low-birth-weight infants: the ARIPI randomized trial. JAMA. 2012;308(14):1443-1451.

11. Dhabangi A, et al. Effect of transfusion of red blood cells with longer vs shorter storage duration on elevated blood lactate levels in children with severe anemia: the TOTAL randomized clinical trial. JAMA. 2015;314(23):2514-2523.

12. Hod EA, et al. Transfusion of red blood cells after prolonged storage produces harmful effects that are mediated by iron and inflammation. Blood. 2010;115(21):4284-4292.

13. Baek JH, et al. Hemoglobin-driven pathophysiology is an in vivo consequence of the red blood cell storage lesion that can be attenuated in guinea pigs by haptoglobin therapy. J Clin Invest. 2012;122(4):1444-1458.

14. Tsai AG, Cabrales P, Intaglietta M. Microvascular perfusion upon exchange transfusion with stored red blood cells in normovolemic anemic conditions. Transfusion. 2004;44(11):1626-1634.

15. Baron DM, et al. Pulmonary hypertension in lambs transfused with stored blood is prevented by breathing nitric oxide. Anesthesiology. 2012;116(3):637-647.

16. Donadee C, et al. Nitric oxide scavenging by red blood cell microparticles and cell-free hemoglobin as a mechanism for the red cell storage lesion. Circulation. 2011;124(4):465-476.

17. Risbano MG, et al. Effects of aged stored autologous red blood cells on human endothelial function. Am J Respir Crit Care Med. 2015;192(10):1223-1233.

18. Prestia K, et al. Transfusion of stored blood impairs host defenses against Gram-negative pathogens in mice. Transfusion. 2014;54(11):2842-2851.

19. Solomon SB, et al. Mortality increases after massive exchange transfusion with older stored blood in canines with experimental pneumonia. Blood. 2013;121(9):1663-1672.

20. Hod EA, et al. Transfusion of human volunteers with older, stored red blood cells produces extravascular hemolysis and circulating non-transferrin-bound iron. Blood. 2011;118(25):6675-6682.

21. Rapido F, et al. Prolonged red cell storage before transfusion increases extravascular hemolysis. J Clin Invest. 2017;127(1):375-382

22. Vermeulen Windsant IC, et al. Blood transfusions increase circulating plasma free hemoglobin levels and plasma nitric oxide consumption: a prospective observational pilot study. Crit Care. 2012;16(3):R95.

23. Shirwany NA, Zou MH. Arterial stiffness: a brief review. Acta Pharmacol Sin. 2010;31(10):1267-1276

24. Lei C, Yu B, Shahid M, Beloiartsev A, Bloch KD, Zapol WM. Inhaled nitric oxide attenuates the adverse effects of transfusing stored syngeneic erythrocytes in mice with endothelial dysfunction after hemorrhagic shock. Anesthesiology. 2012;117(6):1190-1202.

25. Yu B, Lei C, Baron DM, Steinbicker AU, Bloch KD, Zapol WM. Diabetes augments and inhaled nitric oxide prevents the adverse hemodynamic effects of transfusing syngeneic stored blood in mice. Transfusion. 2012;52(7):1410-1422.

26. Berra L, et al. Autologous transfusion of stored red blood cells increases pulmonary artery pressure. Am J Respir Crit Care Med. 2014;190(7):800-807.

27. Bennett-Guerrero E, et al. Evolution of adverse changes in stored RBCs. Proc Natl Acad Sci USA. 2007;104(43):17063-17068.

28. Deuel JW, et al. Hemoglobinuria-related acute kidney injury is driven by intrarenal oxidative reactions triggering a heme toxicity response. Cell Death Dis. 2016;7:e2064.

29. Schaer CA, et al. Haptoglobin preserves vascular nitric oxide signaling during hemolysis. Am J Respir Crit Care Med. 2016;193(10):1111-1122.

30. Reinhart WH, Chien S. Red cell rheology in stomatocyte-echinocyte transformation: roles of cell geometry and cell shape. Blood. 1986;67(4):1110-1118.

31. Hod EA, Spitalnik SL. Harmful effects of transfusion of older stored red blood cells: iron and inflammation. Transfusion. 2011;51(4):881-885.

32. Yu B, et al. Endothelial dysfunction enhances vasoconstriction due to scavenging of nitric oxide by a hemoglobin-based oxygen carrier. Anesthesiology. 2010;112(3):586-594.

33. Kato GJ, Steinberg MH, Gladwin MT. Intravascular hemolysis and the pathophysiology of sickle cell disease. J Clin Invest. 2017;127(3):750-760

34. Fernandez ML, Volek JS. Guinea pigs: a suitable animal model to study lipoprotein metabolism, atherosclerosis and inflammation. Nutr Metab (Lond). 2006;3:17.

35. Mollan TL, et al. $\alpha$-Hemoglobin stabilizing protein (AHSP) markedly decreases the redox potential and reactivity of $\alpha$-subunits of human $\mathrm{HbA}$ with hydrogen peroxide. J Biol Chem. 2013;288(6):4288-4298. 